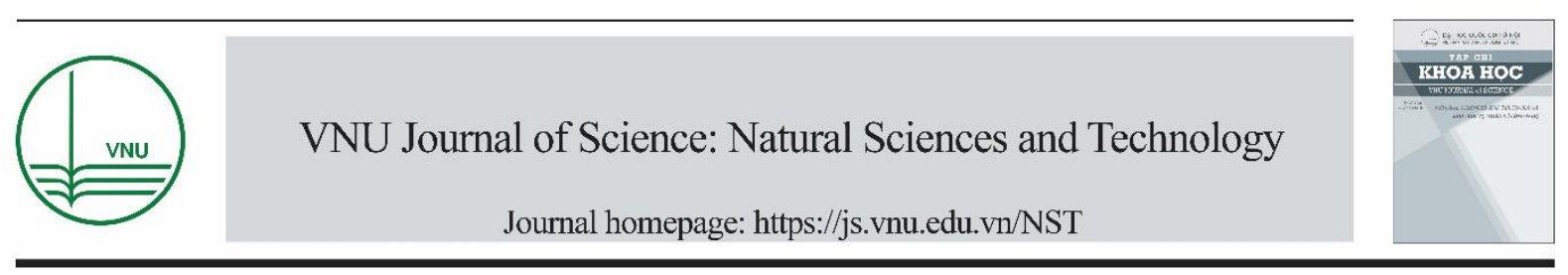

Original Article

\title{
Synthesis of Magnetic Biochar and Their Application for the Treatment of Methylene Blue in Water
}

\author{
Tran Dinh Trinh*, Nguyen Thi Hoai Phuong \\ Faculty of Chemistry, VNU University of Science, Vietnam National University, Hanoi \\ 19 Le Thanh Tong, Hoan Kiem, Hanoi, Vietnam \\ Received 13 August 2019 \\ Revised 22 December 2019; Accepted 13 January 2020
}

\begin{abstract}
Magnetic biochar materials were synthesized by heating rice husk at $500^{\circ} \mathrm{C}$ under nitrogen environment, then fixing iron oxides on biochar surface using hydrothermal method applied to $\mathrm{Fe}(\mathrm{OH})_{2}$ and $\mathrm{Fe}(\mathrm{OH})_{3}$ which were generated from respective precursors $\mathrm{Fe}^{2+}$ and $\mathrm{Fe}^{3+}$ in alkaline environment. The presence of iron oxides on the surface of biochar and the surface characteristics of iron-composite materials were studied with the aid of modern physicochemical analysis techniques (SEM/EDX, BET, FT-IR, XRD). Magnetic biochar materials were relatively porous, with an average spectific surface area of $62.1 \mathrm{~m}^{2}$, an average capillary size of about $17.2 \mathrm{~nm}$. The mixture of iron oxide particles were revealed within the nano scale (about $15 \mathrm{~nm}$ ). The methylene blue adsorption efficiency depended upon the amount of adsorbent, adsorption time, $\mathrm{pH}$ of solution and pollutant concentrations. Specifically, the optimal conditions for maximum adsorption efficiency were as follows: $0.02 \mathrm{~g} / \mathrm{L}$ of magnetic biochar, the adsorption equilibrium time was 3 hours at room temperature, in a solution of $\mathrm{pH} 7$; The efficiency of methylene blue adsorption in optimal conditions reached over $98.82 \%$. The Langmuir and Freundlich isotherm adsorption models all described well the methylene blue adsorption process at room temperature, with the regression coefficients $\mathrm{R}^{2}$ of 95.0 and 90.0 , respectively. The maximum adsorption capacity of methylene blue calculated by Langmuir model was $22.4 \mathrm{mg} / \mathrm{g}$.
\end{abstract}

Keywords: Biochar, mangetic composite, methylene blue, adsorption.

\footnotetext{
${ }^{*}$ Corresponding author.

Email address: trinhtd@ vnu.edu.vn

https://doi.org/10.25073/2588-1140/vnunst.4939
} 


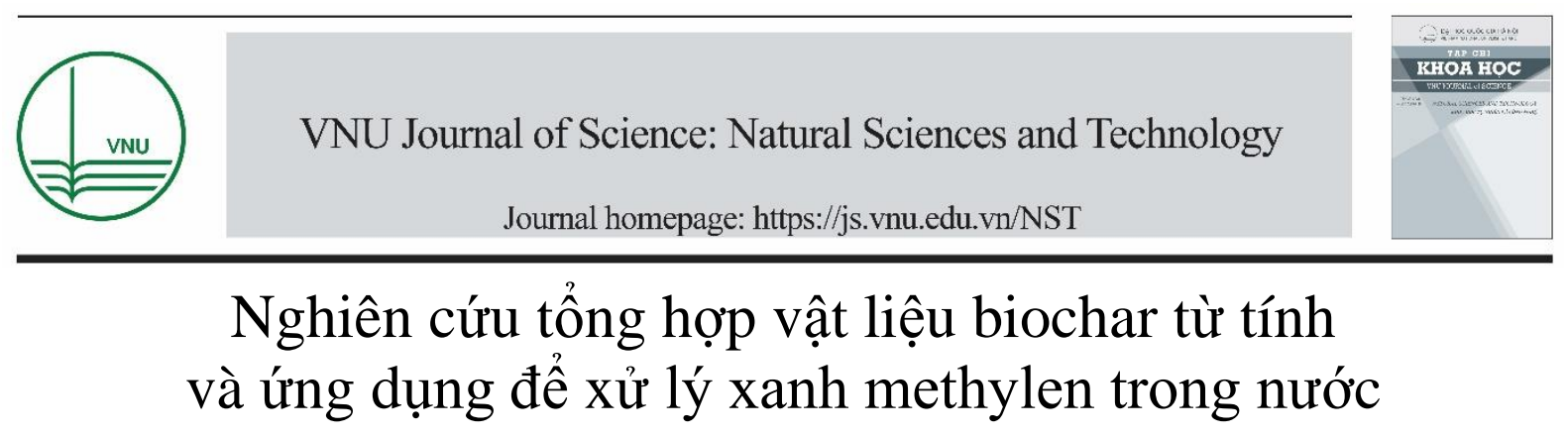

\author{
Trần Đình Trinh*, Nguyễn Thị Hoài Phương
}

Khoa Hóa học, Truờng Đại học Khoa học Tự nhiên, ĐHQGHN, 19 Lê Thánh Tông, Hà Nội, Việt Nam

Nhận ngày 13 tháng 8 năm 2019

Chỉnh sửa ngày 22 tháng 12 năm 2019; Chấp nhận đăng ngày 31 tháng 01 năm 2020

\begin{abstract}
Tóm tắt: Vật liệu biochar từ tính được tổng hợp bằng phương pháp nung vỏ trấu ở $500^{\circ} \mathrm{C}$ trong môi trường nitơ, sau đó cố định các oxit sắt lên bề mặt biochar sử dụng phương pháp thủy nhiệt hỗn hợp $\mathrm{Fe}(\mathrm{OH})_{2}$ và $\mathrm{Fe}(\mathrm{OH})_{3}$ được tạo ra từ tiền chất $\mathrm{Fe}^{2+}$ và $\mathrm{Fe}^{3+}$ trong môi trường kiềm. Sự có mặt của các oxit sắt trên bề mặt biochar và các đặc trưng bề mặt của vật liệu composite biochar-oxit sắt được nghiên cứu bằng các phương pháp SEM/EDX, BET, FT-IR, XRD. Vật liệu biochar từ tính có nhiều lỗ xốp, diện tích bề mặt đạt $62,1 \mathrm{~m}^{2}$, kích thước mao quản trung bình khoảng $17,2 \mathrm{~nm}$, với các hạt oxit sắt có kích thước khoảng $15 \mathrm{~nm}$ phủ trên bề mặt vật liệu. Hiệu suất hấp phụ xanh methylen phụ thuộc vào khối lượng chất hấp phụ, thời gian hấp phụ, $\mathrm{pH}$ của dung dịch và nồng độ chất ô nhiễm. Cụ thể, các điều kiện thích hợp cho quá trình hấp phụ xanh methylen đạt hiệu suất trên 98,82\% như sau: $80 \mathrm{mg} / \mathrm{L}$ vật liệu biochar từ tính, thời gian đạt cân bằng hấp phụ là 3 giờ tại nhiệt độ phòng, trong dung dịch có $\mathrm{pH}=7$; . Các mô hình hấp phụ đẳng nhiệt Langmuir và Freundlich đều mô tả tốt quá trình hấp phụ xanh methylen trong nước tại nhiệt độ phòng với các hệ số hồi quy $\mathrm{R}^{2}$ lần lượt là 95,0 và 90,0 . Tải trọng cực đại của quá trình hấp phụ xanh methylen tính theo mô Hình Langmuir là $\mathrm{q}_{\max }=22,4 \mathrm{mg} / \mathrm{g}$.
\end{abstract}

Tì khóa: Xanh methylen, biochar, composite, oxit sắt từ, hấp phụ.

\section{Mở đầu}

Thuốc nhuộm tổng hợp được sử dụng trong công nghiệp dệt, giấy, cao su, da, nhựa, và ngành công nghiệp mỹ phâm tạo đã phát thải các chất màu, gây ô nhiễm nguồn nước. Xanh methylen là một loại thuốc nhuộm có ý nghĩa quan trọng trong công nghiệp dệt nhưng lại có những tác động độc hại đối với con người, động vật và các hệ thực vật. Những người tiếp xúc với nước thải dệt nhuộm thường mắc các bệnh về $\mathrm{da}$, xanh methylen ngăn cản sự hấp thụ oxy và ánh sáng mặt trời, gây cản trở hô hấp và sự phát triển của các sinh vật dưới nước [1,2].

Đặc điểm nước thải trong ngành dệt và nhuộm

\footnotetext{
*Tác giả liên hệ.

Địa chi email: trinhtd@ vnu.edu.vn

https://doi.org/10.25073/2588-1140/vnunst.4939
} 
là chất rắn lơ lửng, màu, $\mathrm{BOD}$ và $\mathrm{COD}$ cao. Nước thải loại này thường được xử lý bằng tổ hợp các phương pháp như: Cơ học, hóa lý (trung hòa chất thải có tính kiềm hoặc axit mạnh; đông tụ để loại bỏ màu, tạp chất lơ lửng), phương pháp oxy hóa hóa học, điện hóa, sinh học, hấp phụ,... Phương pháp hấp phụ để xử lý phẩm nhuộm thường được sử dụng ở giai đoạn cuối nhằm xử lý triệt để.

Biochar (than sinh học) là than có độ xốp cao được tạo ra từ quá trình nhiệt phân nguyên liệu thô có nguồn gốc từ sinh khối thực vật hoặc phụ phẩm nông nghiệp (thân cây ngô, vỏ đậu phộng, vỏ gỗ, trấu, phân gia súc và phân gia cầm) trong môi trường yếm khí. Các yếu tố chính quyết định đặc tính của biochar là: thành phần vật liệu ban đầu; các yếu tố của quá trình nhiệt phân (nhiệt độ, khí, thời gian phản ứng, chất xúc tác).

Trong quá trình nhiệt phân, ở nhiệt độ thấp xenlulozơ và hemi-xenlulozơ bị mất do bay hơi dẫn tới sự suy giảm về khối lượng. Chất khoáng và bộ khung cacbon vẫn giữ được Hình dạng cấu trúc của vật liệu ban đầu, làm cho than có trạng thái xốp và có diện tích bề mặt lớn [3]. Các nhóm chức bề mặt không những được Hình thành từ phản ứng với oxi trong quá trình nhiệt phân mà còn là kết quả từ phản ứng với các oxit dạng khí như khí nitơ oxit, khí cacbonic,... hay với các dung dịch như axit $\mathrm{HNO}_{3}, \mathrm{H}_{2} \mathrm{O}_{2}, \ldots$ Với các tính chất đã nêu, biochar được sử dung rộng rãi trong nhiều lĩnh vực khác nhau như canh tác nông nghiệp (cải tạo đất, giữ ẩm), xử lý ô nhiễm môi trường (chất hấp phụ, xúc tác, chất mang xúc tác) [3-5].

Biochar là vật liệu hấp phụ tiềm năng vì có các tính chất giống than hoạt tính, đặc biệt việc chế tạo biochar đơn giản hơn than hoạt tính và có thể tạo biochar từ các chất thải có nguồn gốc sinh khối. Điều này đã thúc đẩy các nghiên cứu trong nước và quốc tế về chuyển hóa chất thải thành biochar và ứng dụng trong xử lý môi trường hoặc canh tác nông nghiệp, để đạt đồng thời hai mục tiêu: xử lý chất thải từ sinh khối và xử lý ô nhiễm môi trường, tạo vật liệu cải tạo đất. Tuy nhiên, biochar có kích thước hạt nhỏ, thường khó tách khỏi dung dịch nước sau khi xử lý, do đó đòi hỏi các quá trình lọc, ly tâm để phân tách. Điều này hạn chế việc ứng dụng của chúng trong xử lý nước thải.

Do vậy, để khắc phục những khó khăn trên, trong nghiên cứu này, chúng tôi tập trung nghiên cứu đưa tính chất từ lên vật liệu biochar để nghiên cứu xử lý xanh methylen trong nước. Vật liệu biochar từ tính có thể dễ dàng được tách ra bằng cách sử dụng nam châm hoặc từ trường bên ngoài sau qua trình xử lý nước ô nhiễm.

\section{Thực nghiệm}

\subsection{Hóa chất}

Các hóa chất được sử dụng trong nghiên cứu bao gồm: axit clohidric $(\mathrm{HCl})$, sắt (II) clorua $\left(\mathrm{FeCl}_{2} \cdot 4 \mathrm{H}_{2} \mathrm{O}\right)$, sắt (III) clorua $\left(\mathrm{FeCl}_{3} \cdot 6 \mathrm{H}_{2} \mathrm{O}\right)$, axit nitric $\left(\mathrm{HNO}_{3}\right)$, xanh methylen $\left(\mathrm{C}_{16} \mathrm{H}_{18} \mathrm{ClN}_{3} \mathrm{~S}\right)$ là các hóa chất tinh khiết phân tích của Merck, Đức; natri hidroxit $(\mathrm{NaOH})$, natri nitrat $\left(\mathrm{NaNO}_{3}\right)$ là các hóa chất tinh khiết phân tích của Xilong, Trung Quốc. Vỏ trấu được rửa sạch và sấy khô ở $105^{\circ} \mathrm{C}$ trong 24 giờ trước khi sử dụng trong các thí nghiệm biến tính.

\subsection{Tổng hợp vật liệu}

Biochar chưa biến tính được chế tạo từ vỏ trấu bằng cách sấy vỏ trấu ở $105^{\circ} \mathrm{C}$ trong 24 giờ. Tiếp theo, vỏ trấu được nung trong lò nung ống hãng Carbolite (Anh) trong môi trường nitơ tại $500^{\circ} \mathrm{C}$ trong 1 giờ. Dung dịch $\mathrm{HCl} 0,1 \mathrm{M}$ được thêm vào và khuấy trên máy khuấy từ trong 24 giờ để loại bỏ các thành phần tạp chất. Hỗn hợp được rửa sạch bằng nước cất đến $\mathrm{pH}=7$, sau đó, sấy biochar ở $70^{\circ} \mathrm{C}$ trong 12 giờ, thu được vật liệu biochar chưa biến tính.

Vật liệu biochar từ tính được điều chế bằng cách lấy $11,8 \mathrm{~g} \mathrm{FeCl}_{2} .4 \mathrm{H}_{2} \mathrm{O}+27 \mathrm{~g} \mathrm{FeCl}_{3} \cdot 6 \mathrm{H}_{2} \mathrm{O}$ hòa tan vào $100 \mathrm{~mL}$ nước đề ion. Thêm $\mathrm{NaOH}$ $5 \mathrm{M}$ và khuấy trộn bằng máy khuấy từ ở $80^{\circ} \mathrm{C}$. Thêm $5 \mathrm{~g}$ biochar chưa biến tính và cho từ từ dung dịch $\mathrm{NaOH} 5 \mathrm{M}$ vào hỗn hợp đến $\mathrm{pH}=7$. Tiếp theo, hỗn hợp được quay ly tâm với tốc độ 3000 vòng/phút trong 10 phút để tách lấy chất 
rắn. Phần chất rắn được sấy ở $70^{\circ} \mathrm{C}$ trong 24 giờ để thu được biochar từ tính (biochar- $\mathrm{Fe}_{3} \mathrm{O}_{4}$ ).

\section{3. Đặc trung cấu trúc vật liệu}

Các phương pháp phân tích hóa lý hiện đại được sử dụng để nghiên cứu đặc trưng bề mặt vật liệu như: phương pháp nhiễu xạ tia X (XRD- D8 Advance, hãng Bruker); phương pháp hiển vi điện tử quét SEM (S4700, Hitachi); phương pháp tán xạ năng lượng tia X - EDX (Hitachi S4700 SEM tích hợp phổ tán xạ năng lượng tia X); phương pháp FT-IR (IR Affinity-1S, Shimadzu) và phương pháp Brunauer-Emmett-Teller (BET) trên máy TriStar II Plus, hãng Micromeritics Instrument Corporation, Mỹ.

Xác định điểm đẩng điện của vật liệu: Chuẩn bị $25 \mathrm{~mL}$ dung dịch $\mathrm{NaNO}_{3} 0,1 \mathrm{M}$, điều chỉnh $\mathrm{pH}$ của dung dịch $\mathrm{NaNO}_{3}$ từ $\mathrm{pH}=2$ đến $\mathrm{pH}=12$ bằng các dung dịch $\mathrm{HNO}_{3} 0,1 \mathrm{M}$ và $\mathrm{NaOH} 0,1 \mathrm{M}$. Thêm $0,025 \mathrm{~g}$ biochar từ tính vào $25 \mathrm{~mL}$ dung dịch $\mathrm{NaNO}_{3}$ ở các $\mathrm{pH}$ khác nhau, lắc với tốc độ 150 vòng/phút trong 3 giờ và lọc lấy dung dịch để đo lại giá trị $\mathrm{pH}$ của dung dịch $\left(\mathrm{pH}_{\mathrm{f}}\right)$. Chệnh lệch giữa $\mathrm{pH}$ ban đầu $\left(\mathrm{pH}_{\mathrm{i}}\right)$ và $\mathrm{pH}$ cân bằng $\left(\mathrm{pH}_{\mathrm{f}}\right)$ là $\mathrm{pH}_{\mathrm{i}}-\mathrm{pH}_{\mathrm{f}}=\Delta \mathrm{pH}$, vẽ đồ thị biểu diễn sự phụ thuộc của $\Delta \mathrm{pH}$ vào $\mathrm{pH}$, giao điểm giữa $\Delta \mathrm{pH}$ và trục $\mathrm{pH}$ cho điểm đẳng điện $\left(\mathrm{pH}_{\mathrm{pzc}}\right)$ của vật liệu.

2.4. Nghiên cưu ảnh hương của các điều kiện phản ứng đến hiệu suất xủ lý phẩm nhuộm xanh metylen

\section{Anh hường của thời gian phản ứng}

Cân $0,02 \mathrm{~g}$ vật liệu biochar từ tính vào $25 \mathrm{~mL}$ dung dịch xanh methylen nồng độ $20 \mathrm{mg} / \mathrm{L}$. Tiếp theo, hỗn hợp được lắc liên tục với tốc độ 150 vòng/phút trên máy lắc IKA model KS 260 basic với các khoảng thời gian 30,60,90, 120, 150, 180, 210, 240 phút ở điều kiện nhiệt độ phòng và $\mathrm{pH}$ khoảng 7. Lọc lấy dung dịch và xác định nồng độ xanh methylen còn lại trong dung dịch bằng phương pháp quang, trên máy UV - Vis, model Carry 100, hãng Agilent, Mỹ.

\section{Anh hương của $p H$}

Cân $0,02 \mathrm{~g}$ vật liệu biochar từ tính vào $25 \mathrm{~mL}$ dung dịch xanh methylen nồng độ $20 \mathrm{mg} / \mathrm{L}$, lắc với tốc độ 150 vòng/phút sử dụng máy lắc với thời gian là thời gian đạt cân bằng hấp phụ đã xác định ở trên tại nhiệt độ phòng và $\mathrm{pH}$ của dung dịch được điều chỉnh từ 3 đến 10 . Lọc lấy dung dịch và xác định nồng độ xanh methylen còn lại.

\section{Anh hương của khối luợng chất hấp phu}

Thực hiện các thí nghiệm tương tự như các phần trên với thời gian là thời gian đạt cân bằng hấp phụ, pH tối ưu đã xác định được ở phần trên và điều chỉnh khối lượng biochar từ tính bằng 0,$01 ; 0,02 ; 0,03 ; 0,05$ và $0,1 \mathrm{~g}$.

\section{Ảnh hưởng của nồng độ chất ô nhiễm}

Các thí nghiệm được tiến hành tương tự như phần nghiên cứu ảnh hưởng của thời gian, $\mathrm{pH}$, khối lượng chất hấp phụ; tuy nhiên nồng độ của dung dịch xanh methylen được thay đổi từ 5 đến $40 \mathrm{mg} / \mathrm{L}$ và quá trình hấp phụ được thực hiện đến thời gian đạt cân bằng hấp phụ, $\mathrm{pH}$ tối ưu và lượng chất hấp phụ tối ưu đã xác định được ở các phần nghiên cứu trước.

\subsection{Xác định nồng độ xanh methylen trong nước và tính hiệu suất xử lý}

Xây dụng đường chuẩn xác định nồng độ xanh methylen trong nuoóc: pha các dung dịch xanh methylen với khoảng nồng độ trong khoảng 1 đến $25 \mathrm{mg} / \mathrm{L}$. Đo mật độ quang các dung dịch xanh methylen trên máy UV - VIS (model Carry 100, hãng Agilent, Mỹ) tại bước sóng 665 nm.

Kết quả cho thấy sự phụ thuộc của nồng độ xanh methylen vào độ hấp thụ quang trong dung dịch tuân theo phương trình đường thẳng $\mathrm{y}=$ $0,1803 x+0,364$, với hệ số hồi quy $\mathrm{R}^{2}=0,9995$.

Hiệu suất xử lý của quá trình được xác định theo công thức:

$$
H(\%)=\frac{C_{o}-C_{t}}{C_{o}} \times 100 \%
$$

trong đó: $\boldsymbol{H}$ là hiệu suất xử lý (\%); $\boldsymbol{C}_{\boldsymbol{o}}$ là nồng độ xanh methylen ban đầu $(\mathrm{mg} / \mathrm{L}) ; \boldsymbol{C}_{\boldsymbol{t}}$ là nồng độ xanh methylen tại thời gian phản ứng $\mathrm{t}(\mathrm{mg} / \mathrm{L})$. 


\section{Kết quả và thảo luận}

\section{1. Đặc trung cấu trúc vật liệu}

Kết quả chụp ảnh SEM bề mặt của vật liệu biochar được thể hiện trên Hình 1. Các kết quả thu được cho thấy vật liệu biochar chưa biến tính tương đối xốp, các mao quản nằm song song và xếp sát nhau với đường kính các mao quản khá đồng đều. Trên bề mặt của biochar có xuất hiện tập hợp các hạt nhỏ được xác định là $\mathrm{SiO}_{2}$ từ vỏ trấu thô (phổ EDX Hình 3).

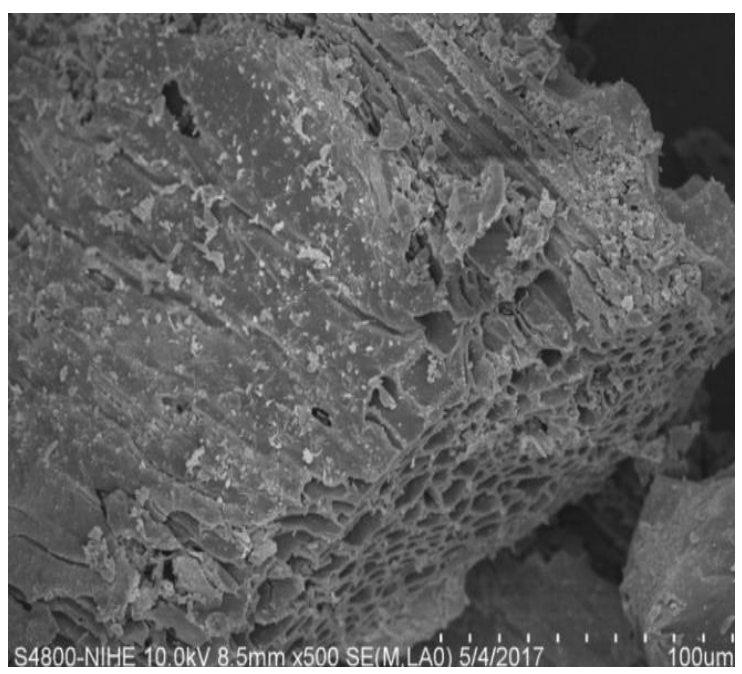

Hình 1a. Ảnh SEM của biochar chưa biến tính.

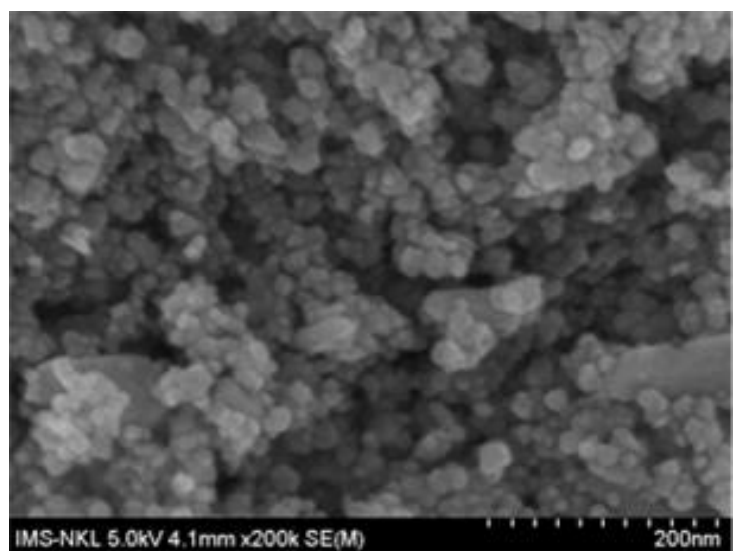

Hình 1b. Ảnh SEM của vật liệu biochar- $-\mathrm{Fe}_{3} \mathrm{O}_{4}$.

Kết quả chụp ảnh SEM bề mặt của vật liệu biochar từ tính cho thấy hầu như không có sự thay đổi về cấu trúc lỗ xốp của biochar, tuy nhiên đã xuất hiện nhiều tập hạt nano trên bề mặt hơn (Hình $1 b$ ). Các hạt này được xác định chủ yếu là $\mathrm{Fe}_{3} \mathrm{O}_{4}$ (Hình 2b).

Kích thước của các hạt oxit kim loại này cùng thành phần hóa học của chúng sẽ được làm rõ trong các phần tiếp theo của bài báo sử dụng các phương pháp phân tích bề mặt như EDX, XRD và IR.

\section{Két quả EDX}

Kết quả phân tích thành phần nguyên tố cho thấy biochar chưa biến tính chứa chủ yếu ba nguyên tố $\mathrm{Si}, \mathrm{O}, \mathrm{C}$ từ $\mathrm{SiO}_{2}$, các nhóm chức $(\mathrm{CO}$, HO-, COO-) và khung cacbon của vật liệu biochar trong khi vật liệu biochar biến tính cho thấy sự xuất hiện của nguyên tố Fe trong hỗn hợp oxit sắt bên cạnh các nguyên tố $\mathrm{C}, \mathrm{O}$ trên khung biochar và $\mathrm{Si}$ trong $\mathrm{SiO}_{2}$ vẫn tồn tại do khó bị hòa tan bởi các axit thông thường (Hình 2).

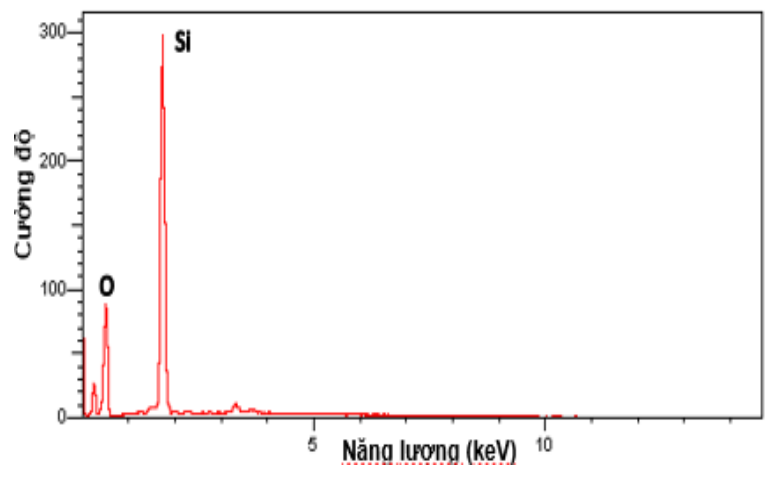

Hình 2a. Phổ EDX của vật liệu biochar chưa biến tính.

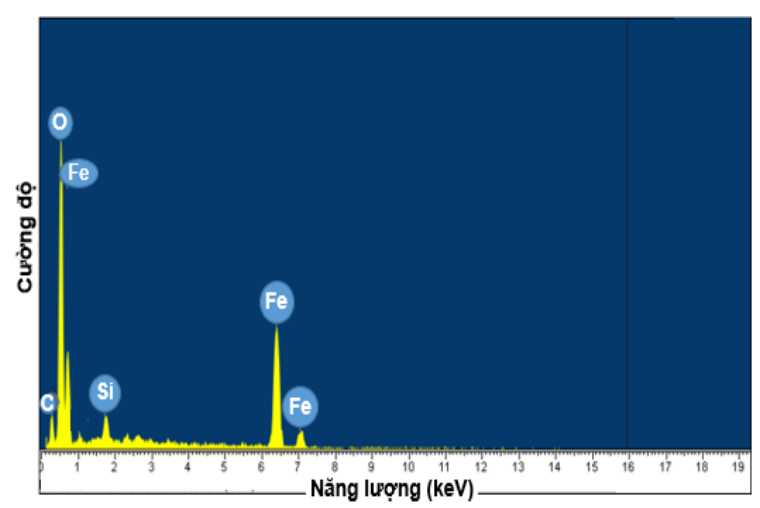

Hình 2b. Phổ EDX của vật liệu biochar- $\mathrm{Fe}_{3} \mathrm{O}_{4}$. 
Cụ thể, vật liệu biochar- $\mathrm{Fe}_{3} \mathrm{O}_{4}$ có tỷ lệ phần trăm khối lượng $\mathrm{O}, \mathrm{C}, \mathrm{Na}, \mathrm{Si}, \mathrm{Fe}$ lần lượt là 38,6 ; $9,4 ; 1,6 ; 1,8 ;$ và 48,6\% (Bảng 1$)$.

Bảng 1. Kết quả phân tích phần trăm nguyên tố trong các vật liệu tính theo EDX.

\begin{tabular}{clllll}
\hline $\begin{array}{l}\text { STT Nguyên } \\
\text { tố }\end{array}$ & \multicolumn{2}{l}{ \% Khối lượng } & \multicolumn{2}{l}{ \% Nguyên tố } \\
\hline & & Biochar & $\mathrm{B}^{-}-\mathrm{Fe}_{3} \mathrm{O}_{4}$ & Biochar & $\mathrm{B}_{-}-\mathrm{Fe}_{3} \mathrm{O}_{4}$ \\
\hline 1 & $\mathrm{O}$ & 43,3 & 38,6 & 45,2 & 57,0 \\
2 & $\mathrm{C}$ & 18,2 & 9,4 & 20,1 & 18,5 \\
3 & $\mathrm{Na}$ & 0,89 & 1,6 & 1,01 & 1,5 \\
4 & $\mathrm{Si}$ & 36,4 & 1,8 & 32,4 & 1,6 \\
5 & $\mathrm{Fe}$ & 1,21 & 48,6 & 1,29 & 20,7 \\
& Tổng & 100 & 100 & 100 & 100 \\
\hline
\end{tabular}

${ }^{*} \mathrm{~B}-\mathrm{Fe}_{3} \mathrm{O}_{4}$ : Biochar- $\mathrm{Fe}_{3} \mathrm{O}_{4}$

Kết quả nhiếu xạ tia X $(X R D)$

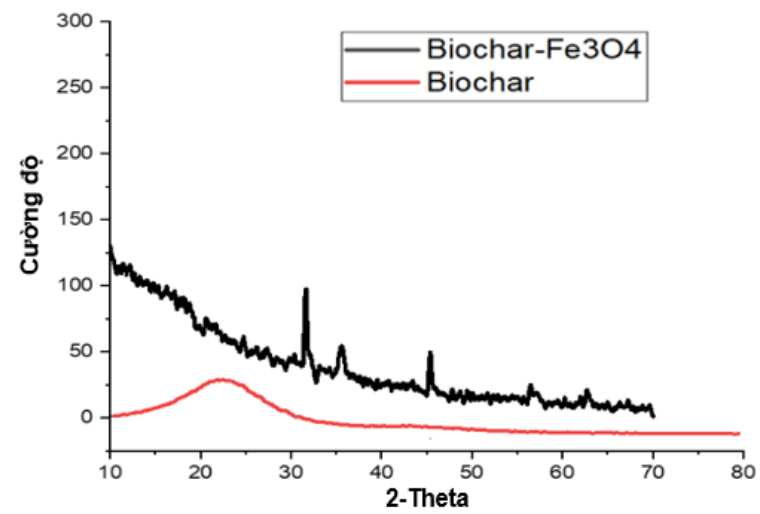

Hình 3. Giản đồ XRD của vật liệu composite biochar- $\mathrm{Fe}_{3} \mathrm{O}_{4}$.

Hình 3 biểu diễn kết quả phân tích nhiễu xạ tia $X$ của vật liệu biochar từ tính. Vật liệu biochar từ tính cho các pic nhiễu xạ đặc trưng tại các góc $2 \theta=21,1 ; 30,3 ; 35,6 ; 45,1,56,9$ và 63 (JCPDS Card No. 19-629). Các pic này lần lượt tương ứng với các mặt phẳng (1 11 1), (2 2 0), (3 11 1), (4 0 0), (4 2 2), (5 11 1), và (4 40 ). Pic có cường độ thấp tại góc $2 \theta=26,2$, tương ứng với mặt phẳng $\left(\begin{array}{lll}0 & 0 & 2\end{array}\right)$ được cho là của các vi tinh thể của cacbon cấu trúc graphit có trong biochar [6]. Kích thước trung bình của các hạt oxit sắt tính theo công thức Debye-Scherrer là khoảng $15 \mathrm{~nm}$.

\section{Kết quả phổ FT - IR}

Kết quả phổ FT-IR của biochar từ tính cho thấy có sự tồn tại của các nhóm chức trên bề mặt biochar và của $\mathrm{Fe}-\mathrm{O}$. Cụ thể, dao động ở quanh vị trí $586 \mathrm{~cm}^{-1}$ là do liên kết $\mathrm{Fe}-\mathrm{O}$ [7], trong khi dao động tại bước sóng $3434 \mathrm{~cm}^{-1}$ là dao động của nhóm $\mathrm{O}-\mathrm{H}$ trên bề mặt biochar và hơi nước hấp phụ trên bề mặt vật liệu.

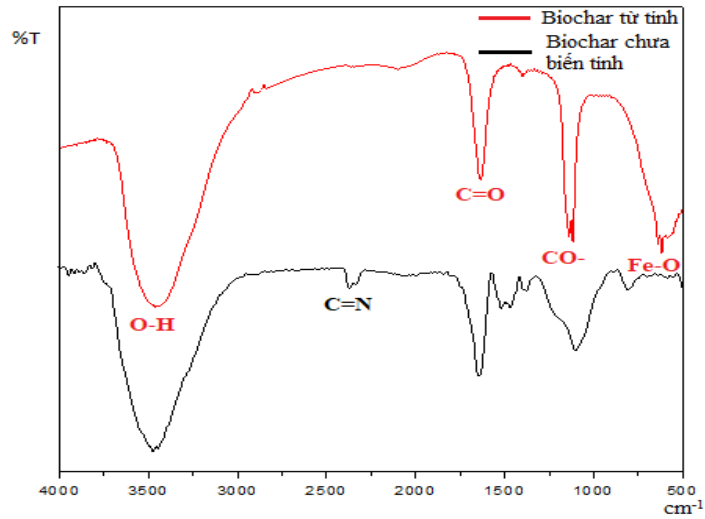

Hình 4. Phổ FT - IR của vật liệu biochar từ tính và biochar chưa biến tính.

Liên kết este $\mathrm{C}=\mathrm{O}$, vòng thơm $\mathrm{CO}$ - có đặc trưng dao động tại bước sóng $1635 \mathrm{~cm}^{-1}$ và 1101 $\mathrm{cm}^{-1}$, liên kết $\mathrm{C}=\mathrm{N}$ có đặc trưng dao động tại bước sóng $2360 \mathrm{~cm}^{-1}[8,9]$.

Việc Hình thành oxit $\mathrm{Fe}_{3} \mathrm{O}_{4}$ và liên kết của nó với bề mặt biochar đã được nghiên cứu và thảo luận trong một số nghiên cứu trước [10,11]. Trước tiên, bề mặt biochar sẽ tạo các tâm giúp Hình thành các oxit trong quá trình xử lý hỗ hợp $\mathrm{Fe}^{2+}$ và $\mathrm{Fe}^{3+}$ bằng dung dịch $\mathrm{NaOH}$. Trong quá trình này có sự hình thành các liên kết giữa các oxit sắt và bề mặt biochar thông qua các nhóm chức $-\mathrm{OH}$ và $-\mathrm{COOH}$ thông qua cầu nối oxi, sau đó các oxit $\mathrm{FeO}$ và $\mathrm{Fe}_{2} \mathrm{O}_{3}$ bị giữ lại trên bề mặt của biochar. Liên kết giữa biochar và oxit sắt từ có thể được biểu diễn như sau biochar-O$\mathrm{FeO} / \mathrm{Fe}_{2} \mathrm{O}_{3}$. Liên kết giữa oxi trên bề mặt của biochar với các oxit sắt kết hợp với hiệu ứng columb và hiệu ứng khóa cơ học giữa các pha và liên kết hydro (ví dụ giữa $\mathrm{Fe}-\mathrm{OH}$ trên bề mặt các oxit sắt và $\mathrm{C}-\mathrm{OH}$ trên bề mặt biochar) càng làm bền liên kết giữa oxit sắt từ và biochar [11]. 


\subsection{Kết quả đo diện tích bề mặt riêng theo BET}

Kết quả nghiên cứu diện tích bề mặt riêng của vật liệu biochar và biochar- $\mathrm{Fe}_{3} \mathrm{O}_{4}$ theo phương pháp BET cho thấy, đường đằng nhiệt hấp phụ-khử hấp phụ nitơ của hai loại vật liệu này thuộc kiểu thứ $\mathrm{V}$. Vật liệu biochar- $\mathrm{Fe}_{3} \mathrm{O}_{4}$ có vòng trễ dạng $\mathrm{H} 3$ (theo phân loại của IUPAC), đặc trưng cho vật liệu có mao quản trung bình và tương ứng với sự phân bố kích thước mao quản Barret-Joyner-Halenda (BJH). Vật liệu biochar có vòng trễ, đặc trưng cho vật liệu mao quản hình khe (Hình 5a).

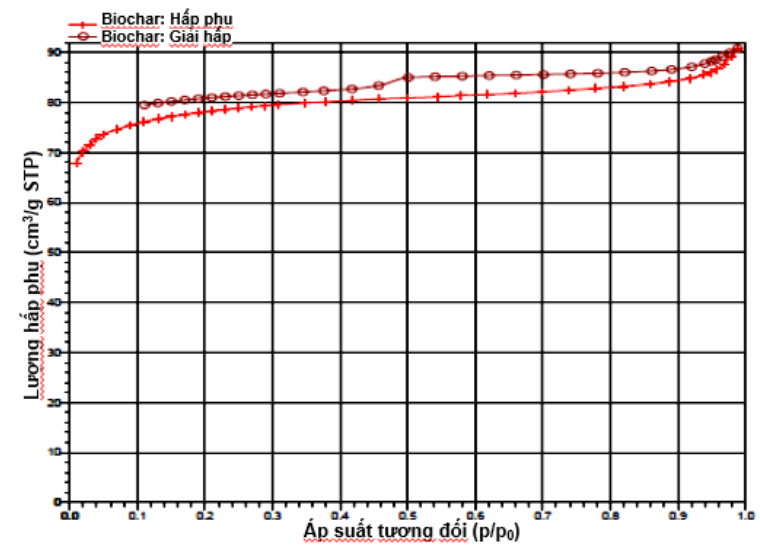

Hình 5a. Đường cong hấp phụ-giải hấp phụ $\mathrm{N}_{2}$ tại $77 \mathrm{~K}$ của vật liệu biochar chưa biến tính.

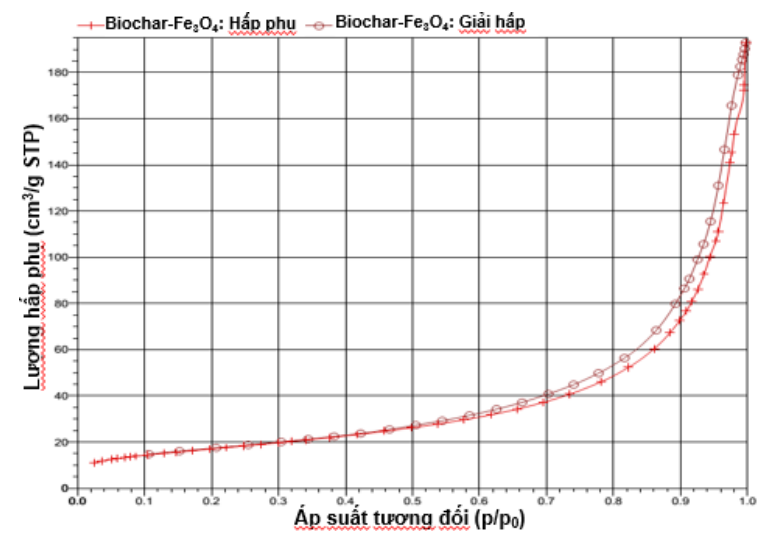

Hình $5 b$. Đường cong hấp phụ-giải hấp phụ $\mathrm{N}_{2}$ tại $77 \mathrm{~K}$ của vật liệu biochar- $\mathrm{Fe}_{3} \mathrm{O}_{4}$.

Hình $5 b$ cũng cho thấy, đường giải hấp của biochar chưa biến tính và biochar- $\mathrm{Fe}_{3} \mathrm{O}_{4}$ tương đối mịn, trong đó, đặc biệt vật liệu biochar- $\mathrm{Fe}_{3} \mathrm{O}_{4}$ có đường cong giải hấp phụ- khử hấp phụ đẳng nhiệt bắt đầu ngưng tụ ở áp suất tương đối $\mathrm{P} / \mathrm{P}_{0}$ trong khoảng rộng $(0,1-1,0)$, chứng tỏ vật liệu có đường kính mao quản tương đối lớn. Trong khi đó vật liệu biochar chưa biên tính có đường giải hấp không có sự đóng vòng kín tại áp xuất tương đối $\mathrm{P} / \mathrm{P}_{0}$ trong khoảng $<0,1$ điề̀u này cho thấy ngoài hạt có đường kính mao quản lớn trong vật liệu còn có xuất hiện các đường kính mao quản nhỏ (vi mao quản). Kết quả đo diện tích bề mặt riêng và kích thước mao quản của hai vật liệu như sau: biochar chưa biến tính có diện tích bề mặt riêng là $138 \mathrm{~m}^{2} / \mathrm{g}$; kích thước mao quản từ $1,7 \mathrm{~nm}$ đến $300 \mathrm{~nm}$; thể tích mao quản: 0,20 $\mathrm{cm}^{3} / \mathrm{g}$, đường kính mao quả trung bình: $5,5 \mathrm{~nm}$. Vật liệu biochar- $\mathrm{Fe}_{3} \mathrm{O}_{4}$ có diện tích bề mặt riêng đạt $62,1 \mathrm{~m}^{2} / \mathrm{g}$; kích thước mao quản từ $1,7 \mathrm{~nm}$ đển $300 \mathrm{~nm}$; thể tích mao quản: $0,024 \mathrm{~cm}^{3} / \mathrm{g}$, đường kính mao quả trung bình đạt 17,2 nm.

\subsection{Kết quả xác định điểm đẳng điện}

Kết quả thu được chỉ ra rằng, điểm đẳng điện của vật liệu biochar từ tính là tại pH7. Như vậy, bề mặt vật liệu sẽ tích điện dương trong các dung dịch có $\mathrm{pH}$ nhỏ hơn 7 và tích điện âm trong các dung dịch có pH lớn hơn 7.

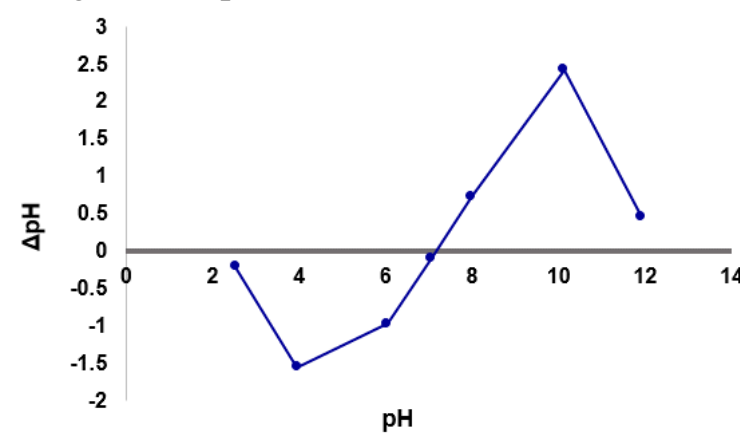

Hình 6. Đồ thị xác định điểm đẳng điện của vật liệu biochar- $\mathrm{Fe}_{3} \mathrm{O}_{4}$.

Việc xác định điểm đẳng điện của vật liệu composite đã tổng hợp cho phép giải thích ảnh hưởng của $\mathrm{pH}$ đến hiệu quả xử lý xanh methylen trong phần tiếp theo của bài báo.

Do hạn chế về điều kiện thực nghiệm, trong nghiên cứu này các thông số về Độ từ hóa (magnetization) như Lực kháng từ $\mathrm{Hc}$ (coercivity), Từ trễ (magnetic hysteresis) của vật liệu biochar từ tính không được xác định. Tuy 
nhiên, cũng như nhiều công bố quốc tế khác, vật liệu biochar từ tính sau khi dùng để xử lý xanh methylen có thể được tách ra khỏi dung dịch để thu hồi, tái sinh bằng nam châm (Mục 3.4.4) thì cũng chứng tỏ rằng vật liệu có từ tính và đáp ứng được mục tiêu của nghiên cứu.

\subsection{Kết quả quá trình xủ lý xanh methylen}

\subsubsection{Anh hường của thời gian}

Hiệu quả xử lý xanh methylen trong dung dịch có nồng độ đầu $20 \mathrm{mg} / \mathrm{L}$ bằng vật liệu biochar từ tính được xác định ở các thời gian phản ứng khác nhau. Có thể thấy rằng khả năng hấp phụ xanh methylen của vật liệu đạt 98,82\% sau 3 giờ. Khi tăng thời gian phản ứng, nồng độ xanh methylen trong dung dịch hầu như không đổi, gợi ý rằng thời gian đạt cân bằng hấp phụ là 3 giờ.

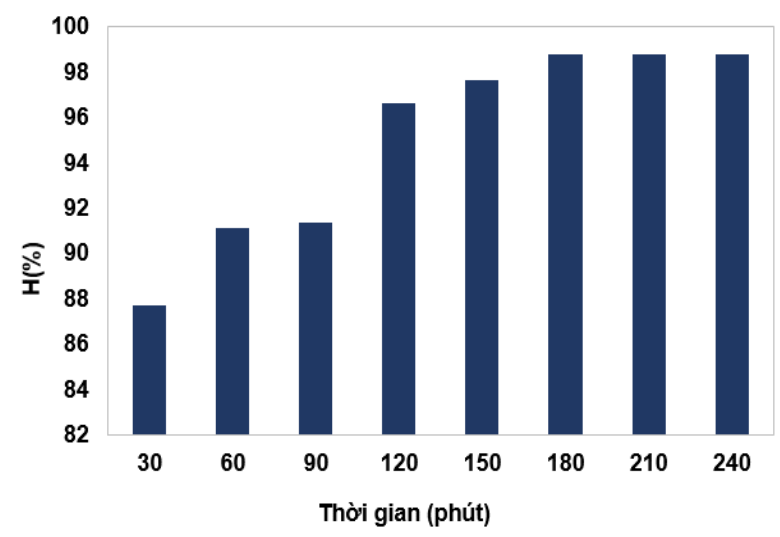

Hình 7. Ảnh hưởng của thời gian đến hiệu suất hấp phụ xanh methylen trên vật liệu biochar- $\mathrm{Fe}_{3} \mathrm{O}_{4}$.

\subsubsection{Anh hướng của $p H$}

Có thể nhận thấy rằng, hiệu quả xử lý xanh methylen tăng dần khi $\mathrm{pH}$ tăng từ 3 đến 8 . Khi $\mathrm{pH}$ của dung dịch thay đổi từ 8 đến 10 thì hiệu suất xử lý thay đổi không đáng kể. Cụ thể, $\mathrm{H}(\%)$ tăng từ $77,8 \%$ tại $\mathrm{pH} 3$ lên $95,5 \%$ tại $\mathrm{pH} 7$. Khi tiếp tục tăng $\mathrm{pH}$ của dung dịch lên giá trị 8 thì $\mathrm{H}(\%)$ tăng lên $98,0 \%$ và hầu như không tăng khi pH của dung dịch tiếp tục tăng lên 10 .

Điều này được giải thích do bề mặt vật liệu biochar- $\mathrm{Fe}_{3} \mathrm{O}_{4}$ mang điện tích dương trong các dung dịch có $\mathrm{pH}<7$ (điểm đẳng điện: $\mathrm{pH}_{\mathrm{pzc}}$ ) trong khi xanh methylen là phẩm nhuộm cation nên sẽ bị đẩy ra khỏi bề mặt vật liệu biochar- $\mathrm{Fe}_{3} \mathrm{O}_{4}$. Bên cạnh đó, trong các dung dịch có $\mathrm{pH}$ thấp hơn việc hấp phụ xanh methylen còn bị cạnh tranh bởi các ion $\mathrm{H}^{+}$trong dung dịch vốn có đường kính rất nhỏ hơn và linh động hơn rất nhiều so với phân tử xanh methylen. Kết quả là, khi $\mathrm{pH}$ của dung dịch càng nhỏ (môi trường càng axit) thì hiệu quả xử lý xanh methylen càng thấp. Một cách tương tự, khi $\mathrm{pH}$ của dụng dịch lớn hơn 7 sẽ thúc đẩy việc hấp phụ xanh methylen lên bề mặt biochar$\mathrm{Fe}_{3} \mathrm{O}_{4}$ thông qua cơ chế tương tác tĩnh điện. Tuy nhiên, khi tăng $\mathrm{pH}$ hơn nữa sẽ có một lượng lớn ion $\mathrm{HO}^{-}$trong dung dịch sẽ tham gia hấp phụ cạnh tranh với phân tử xanh methylen làm cho hiệu quả xử lý không tăng mà thậm chí còn giảm.

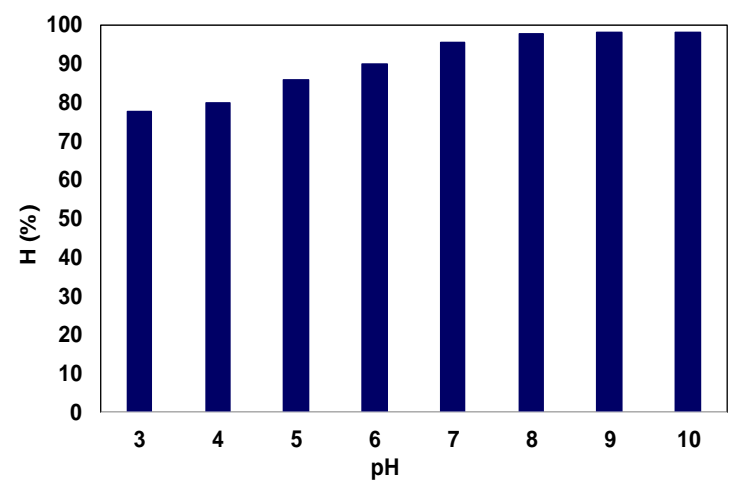

Hình 8. Ảnh hưởng của $\mathrm{pH}$ đến dung lượng hấp phụ xanh methylen của vật liệu biochar từ tính.

\subsubsection{Anh hưởng của khối luợng chất hấp phu}

Kết quả trên Hình 9 chỉ ra rằng, hiệu suất hấp phụ đã tăng từ $92,44 \%$ đến $97,82 \%$ khi khối lượng của biochar từ tính tăng từ $0,01(\mathrm{~g})$ lên $0,02(\mathrm{~g})$. Khi tiếp tục tăng khối lượng biochar$\mathrm{Fe}_{3} \mathrm{O}_{4}$ hiệu suất hấp phụ xanh methylen không những không tăng mà giảm về $94,2 \%$ khi tăng lượng chất hấp phụ lên giá trị $0,1(\mathrm{~g})$.

Điều này có thể được giải thích là do khi lượng chất hấp phụ quá nhỏ, tổng diện tích bề mặt của biochar- $-\mathrm{Fe}_{3} \mathrm{O}_{4}$ rất thấp, không đủ hấp phụ toàn bộ xanh methylen, trong khi lượng chât hấp phụ quá cao sẽ dẫn đến sự co cụm các hạt vật liệu với nhau làm giảm diện tích bề mặt từ đó giảm hiệu quả xử lý [12]. Lượng chất hấp phụ tối ưu cho quá trình xử lý là $20 \mathrm{mg} / 25 \mathrm{~mL}$ dung dịch, hay $80 \mathrm{mg} / \mathrm{L}$. 


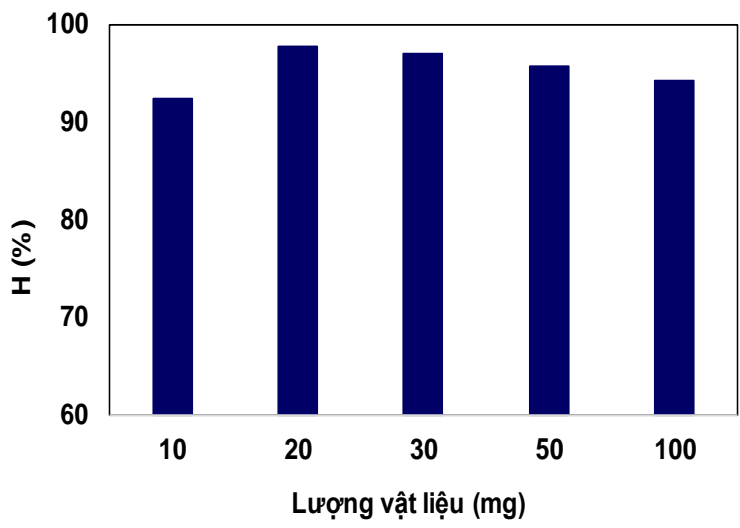

Hình 9. Ảnh hưởng của khối lượng chất hấp phụ đến dung lượng hấp phụ xanh methylen của vật liệu biochar từ tính.

\subsection{4. Ảnh hưởng của nồng độ đầu}

Kết quả thu được chỉ ra rằng khi nồng độ đầu của xanh methylen tăng, hiệu quả xử lý của vật liệu biochar- $\mathrm{Fe}_{3} \mathrm{O}_{4}$ cũng tăng theo (Hình 10). Từ các kết quả thu được chúng tôi tiến hành mô tả quá trình hấp phụ xanh methylen trên vật liệu biochar từ tính sử dụng các mô hình hấp phụ đẳng nhiệt thông dụng là các mô hình Langmuir và Freundlich.

Các kết quả thu được cho thấy, mô hình hấp phụ đẳng nhiệt Langmuir mô tả quá trình hấp phụ xanh methylen bởi vật liệu biochar từ tính tốt hơn mô hình Freundlich. Điều này được thể hiện ở hệ số hồi quy của mô hình Langmuir $(0,95)$ cao hơn so với mô hình Freundlich $(0,90)$. Tải trọng hấp phụ cực đại tính theo mô hình Langmuir đạt $22,4 \mathrm{mg} / \mathrm{g}$ vật liệu.

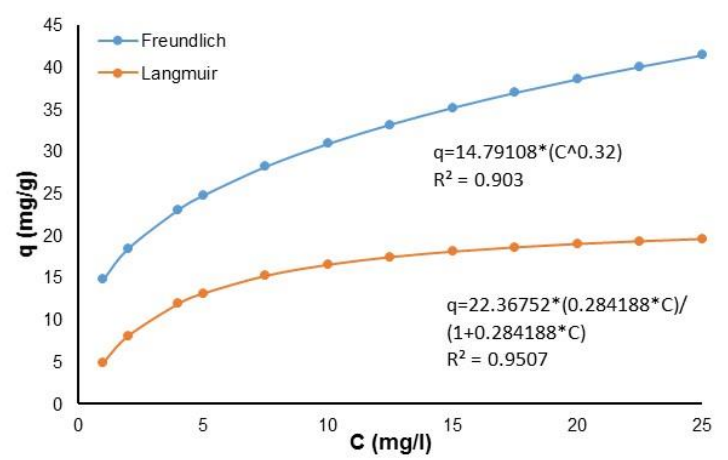

Hình 10. Đường cong phụ thuộc của tải trọng hấp phụ vào nồng độ còn lại của xanh methylen.
Việc thu hồi, tách vật liệu biochar- $\mathrm{Fe}_{3} \mathrm{O}_{4} \mathrm{sau}$ mỗi thí nghiệm xử lý xanh methylen đều có thể được thực hiện một cách dễ dàng bằng cách sử dụng Nam châm đặt phía ngoài dung dịch như trong Hình 11.

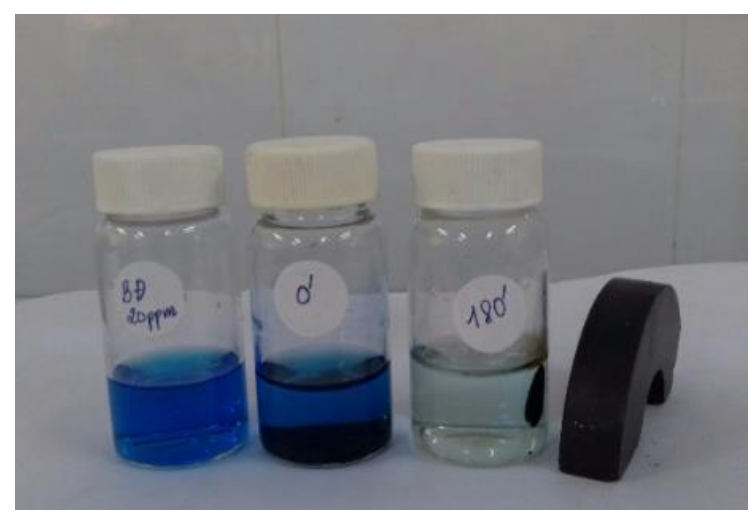

Hình 11. Tách vật liệu hấp phụ và chất ô nhiễm ra khỏi dung dịch sau khi xử lý bằng Nam châm.

Các thí nghiệm về độ bền và khả năng tái sử dụng của vật liệu biochar từ tính đã được nghiên cứu trong một số báo cáo trước đây. Cụ thể, trong nghiên cứu hấp phụ phenanthren trong nước bởi biochar từ tính, Guo và cộng sự (2018) đã chứng minh được rằng oxit sắt từ không bị hòa tan tại các giá trị $\mathrm{pH}$ lớn hơn hoặc bằng 6 trong khi chỉ một lượng nhỏ ion sắt đi vào dung dịch khi dung dịch có tính axit mạnh tại $\mathrm{pH}=2$ [13]. Tương tự, Xin và nhóm nghiên cứu (2016) đã chỉ ra rằng hiệu suất hấp phụ ion $\mathrm{Cr}^{6+}$ trong dung dịch bởi biochar từ tính hầu như không giảm sau bốn lần tái sinh (hiệu suất khoảng $80 \%$ ). Nhóm nghiên cứu cũng chứng minh $\mathrm{Fe}_{3} \mathrm{O}_{4}$ liên kết với bề mặt biochar và không bị tan vào dung dịch [14]. Như vậy, có thể cho rằng quá trình hấp phụ xanh metylen bởi biochar từ tính trong các dung dịch trung tính như ở nghiên cứu hiện tại sẽ có rất ít hoặc không có sự hòa tan oxit $\mathrm{Fe}_{3} \mathrm{O}_{4}$ thành các ion $\mathrm{Fe}^{2+}$ và $\mathrm{Fe}^{3+}$ vào dung dịch.

\section{Kết luận}

Tổng hợp thành công vật liệu biochar từ tính từ vỏ trấu và tiền chất là hồn hợp muối sắt trong môi trường nitơ tại nhiệt độ $500^{\circ} \mathrm{C}$ trong 1 giờ. 
Đặc trưng cấu trúc vật liệu cho thấy sự hình thành các tinh thể oxit sắt có kích thước nano và các hạt có kích thước đồng đều (khoảng $15 \mathrm{~nm}$ ). Sự xuất hiện của các nguyên tố $\mathrm{C}, \mathrm{O}, \mathrm{Si}, \mathrm{Fe}, \mathrm{Na}$ và các nhóm chức $\mathrm{HO}-, \mathrm{C}=\mathrm{C}, \mathrm{C}=\mathrm{O}, \mathrm{CO}-, \mathrm{C}-\mathrm{O}-$ $\mathrm{C}$ lần lượt được chứng minh bởi các phương pháp phân tích EDX và IR. Diện tích bề mặt của vật liệu biochar- $\mathrm{Fe}_{3} \mathrm{O}_{4}$ thấp hơn so với vật liệu biochar chưa biến tính $\left(62,1 \mathrm{~m}^{2} / \mathrm{g}\right.$ so với 138 $\mathrm{m}^{2} / \mathrm{g}$ ) trong khi đó đường kính mao quản trung bình của biochar biến tính $(17,2 \mathrm{~nm})$ lớn hơn so với vật liệu biochar chưa biến tính $(5,5 \mathrm{~nm})$.

Quá trình xử lý xanh methylen trong nước cho thấy thời gian đạt cân bằng hấp phụ là 3 giờ. $\mathrm{pH}$ của dung dịch cho hiệu quả xử lý xanh methylen tốt nhất là ở $\mathrm{pH}$ trung tính do gần với điểm đẳng điện của vật liệu biochar- $\mathrm{Fe}_{3} \mathrm{O}_{4}$ và do xanh methylen là thuốc nhuộm cation. Lượng chất hấp phụ tối ưu là $80 \mathrm{mg} / \mathrm{L}$ được giải thích do khi tăng lượng vật liệu biochar- $\mathrm{Fe}_{3} \mathrm{O}_{4}$ làm tăng khả năng hình thành các tập hợp hạt vật liệu từ đó giảm diện tích bề mặt dành cho hấp phụ trong khi lượng vật liệu ít thì diện tích bề mặt riêng thấp làm cho hiệu suất xử lý không cao. Mô hình hấp phụ đẳng nhiệt Langmuir mô tả chính xác quá trình hấp phụ xanh methylen trong dung dịch bởi vật liệu composite biochar- $\mathrm{Fe}_{3} \mathrm{O}_{4}$, được thể hiện ở hệ số hồi quy là 0,950 . Tải trọng hấp phụ cực đại tính theo mô hình Langmuir là 22,4 $\mathrm{mg} / \mathrm{g}$.

\section{Lời cảm ơn}

Công trình này được hoàn thành với sự hỗ trợ kinh phí của đề tài Nghiên cứu Ứng dụng và Phát triển Công nghệ cấp Quốc gia mã số BĐKH.02/16-20. Các tác giả cảm ơn sự hỗ trợ trang thiết bị thuộc chương trình ROHAN Catalysis và OEPAC tại Phòng thí nghiệm trọng điểm Vật liệu tiên tiến ứng dụng trong Phát triển xanh, ĐHQGHN.

\section{Tài liệu tham khảo}

[1] A.F. Baybars, Ö. Cengiz, K. Mustafa, Cationic Dye (Methylene Blue) Removal from Aqueous Solution by Montmorillonite, Bulletin of the
Korean Chemical Society 33 (2012) 3184-3190. https://doi.org/10.5012/bkcs.2012.33.10.3184.

[2] Md. Juned, K. Ahmed, M. Ahmaruzzaman, A facile synthesis of $\mathrm{Fe}_{3} \mathrm{O}_{4}$ - charcoal composite for the sorption of a hazardous dye from aquatic environment, Journal of Environmental Management 163(2015) 163-173. https://doi.org/ 10.1016/j.jenvman.2015.08.011.

[3] J.S. Cha, S.H. Park, S.-C. Jung, C. Ryu, J.-K. Jeon, M.- C. Shin, Production and Utilization of Biochar: A Review, Journal of Industrial and Engineering Chemistry 40 (2016) 1-15. https:// doi.org/10.1016/j.jiec.2016.06.002.

[4] J.M. Novak, W.J. Busscher, D.W. Watts, D.A. Laird, M.A. Ahmedna, M.A.S. Niandou, Shortterm $\mathrm{CO}_{2}$ mineralization after additions of biochar and switchgrass, Geoderma 154 (2010) 281-288. https://doi.org/10.1016/j.geoderma.2009. 10.014

[5] Y. Zhang, Z. Li, I.B. Mahmood, Recovery of $\mathrm{NH}^{4+}$ by corn cob produced biochars and its potential application as soil conditioner, Journal of Environmental Science and Engineering 8 (2014) 825-834. https://doi.org/10.1007/s11783014-0682-9.

[6] Md.J.K. Ahmed, M. Ahmaruzzaman, R.A. Reza, Lignocellulosic-derived modified agricultural waste: development, characterisation and implementation in sequestering pyridine from aqueous solutions, Journal of Colloid and Interface Science 428 (2014) 222-234. https:// doi.org/10.1016/j.jcis.2014.04.049.

[7] Y.-R. Zhang, S.-Q. Wang, S.-L. Shen, B.-X. Zhao, A novel water treatment magnetic nanomaterial for removal of anionic and cationic dyes under severe condition, Chemical Engineering Journal 233 (2013) 258-264. https: //doi.org/10.1016/j.cej.2013.07.009.

[8] L. Ai, C. Zhang, F. Liao, Y. Wang, M. Li, L. Meng, J. Jiang, Removal of methylene blue from aqueous solution with magnetite loaded multiwall carbon nano tube: kinetics, isotherm and mechanism analysis, Journal of Hazardous Materials 198 (2011) 282-290. https://doi.org/10. 1016/j.jhazmat.2011.10.041.

[9] R. Li, J.J. Wang, B. Zhou, Z. Zhang, S. Liu, S. Lei, R. Xiao, Simultaneous capture removal of phosphate, ammonium and organic substances by $\mathrm{MgO}$ impregnated biochar and its potential use in swine wastewater treatment, Journal of Cleaner Production 147 (2017) 96-107. https://doi.org/ 10.1016/j.jclepro.2017.01.069.

[10] C-D. Dong., C-W. Chen., C-M. Kao., C-C. Chien and C-M. Hung. Wood-Biochar-Supported 
Magnetite Nanoparticles for Remediation of PAH-Contaminated Estuary Sediment, Catalysts 8 (2018) 1-13. https://doi.org/10.3390/catal8020073.

[11] D. Mohan., H. Kumar, A. Saraswat., M. Alexandre - Franco., C.U. Pittman Jr, Cadmium and Lead Remediation using Magnetic Oak Wood and Oak Bark Fast Pyrolysis Bio-chars, Chemical Engineering Journal 236 (2014) 513-528. https:// doi.org/10.1016/j.cej.2013.09.057.

[12] C. Baoliang, C. Zaiming, L. Shaofang, A novel magnetic biochar efficiently sorbs organic pollutants and phosphate, Bioresource
Technology 102 (2011) 716-723. https://doi.org/ 10.1016/j.biortech.2010.08.067.

[13] W. Guo., S. Wang., Y. Wang., S. Lu., Y. Gao. Sorptive removal of phenanthrene from aqueous solutions using magnetic and non-magnetic rice husk-derived biochars, R. Soc. open sci. 5 (2018) 1-11. http://dx.doi.org/10.1098/rsos.172382.

[14] O. Xin, H. Yitong, C. X. and C. Jiawei, Magnetic biochar combining adsorption and separation recycle for removal of chromium in aqueous solution, Water Science \& Technology 75 (2016) 1175 -1184. https://doi.org/10.2166/wst.2016.610. 